\title{
A model of population migration based on Minimum cost and maximum flow
}

\author{
Dan Chen \\ School of North China Electric Power University, Baoding 071000, China; \\ 1241341732@qq.com
}

Keywords: AHP, Refugees Crisis, Dynamic analysis, Minimal Cost, Maximal Flow.

\begin{abstract}
In order to measure the refugee crisis, we adopt analytic hierarchy process(AHP), taking individuals themselves. Then we got a criterion to measure the refugee crisis. The results of the analytic hierarchy process are consistent with the actual situation, which shows that the selected parameters are relatively reasonable. To determine the optimal flow model, we use the min-cost max-flow algorithm to develop a reasonable flow of refugees. We set the target to be the minimum amount of death, the fastest to move and the most balanced of routes. Three strategies can be set correspondingly. The first strategy is regarded as the best, in which the number of casualties is minimal and other . Multi-objective optimization dynamic programming model was used to realize the best allocation of resources in different regions. We studied the change of three resources, accommodation, health care and food. Our result shows that the feed is the biggest consumption, followed by the capacity to house and protect. Therefore, food needs priority. When the non-governmental organizations and other refugee destinations are added, the tendency of the change becomes gentle.We considered the impact of exogenous events, such as terrorist attack. The number of entry points and the routes they must take may be changed completely. With the change of the capacity of countries, the best migration strategy will change accordingly.
\end{abstract}

\section{Introduction}

In recent years, a large number of refugees swarmed into Europe from the Middle East and Africa countries to seek asylum. This makes the European ushered in the largest refugee crisis since World War II. Foreign refugees continue to impact on Europe, intensified. Refugees into European countries are an important part of the global refugee, mainly from the West Asia and North Africa, especially in Syria and Afghanistan.

Refugee crisis is one of the important issues facing the international community today. It not only affects the country that produces the refugee, but also has great influence on the region and even the whole world. Nowadays, with the development of globalization, the refugee problem is no longer a country, a region, but a global problem that the international community faces.

\section{The Optimal Refugee Migration Model}

\section{The model analysis.}

Due to the different origin of refugees, we adopt the min-cost max-flow algorithm to arrange the route for refugees' migration reasonably. The departure of refugees and various of entry points in Europe as the vertexes, refugee migration routes as the directed arcs, a empowered communication directed graph $\mathrm{G}=(\mathrm{V}, \mathrm{E}, \mathrm{C})$ is established, where $\mathrm{V}$ is the set of vertices, $\mathrm{E}$ is the set of directed edges, $\mathrm{C}$ is the capacity of the arc. We consider the origin of the refugees as Vs, and it can be seen that Vs is not only. In addition, the flows of refugee flow freely between the points, just restricted by geographical and martial factors. And then, with comprehensive assessment of such factors as the safety, cost and the number of entry points, $\mathrm{C}$ is calculated. At last, on account of the number and position of Vt correspondingly changing, they need to be considered separately. 
Model Establishing and Solving.

The establishment of Class hierarchy model

Migration routes of refugees are generally divided into six routes, hence there exist six evaluation factors: the individuals themselves, the routes they must take, the types of transportation, the countries' capacity, the number of entry points and the available resources. Based on the optimal route evaluation factors and six routes, we can establish a hierarchical structure model as follows:

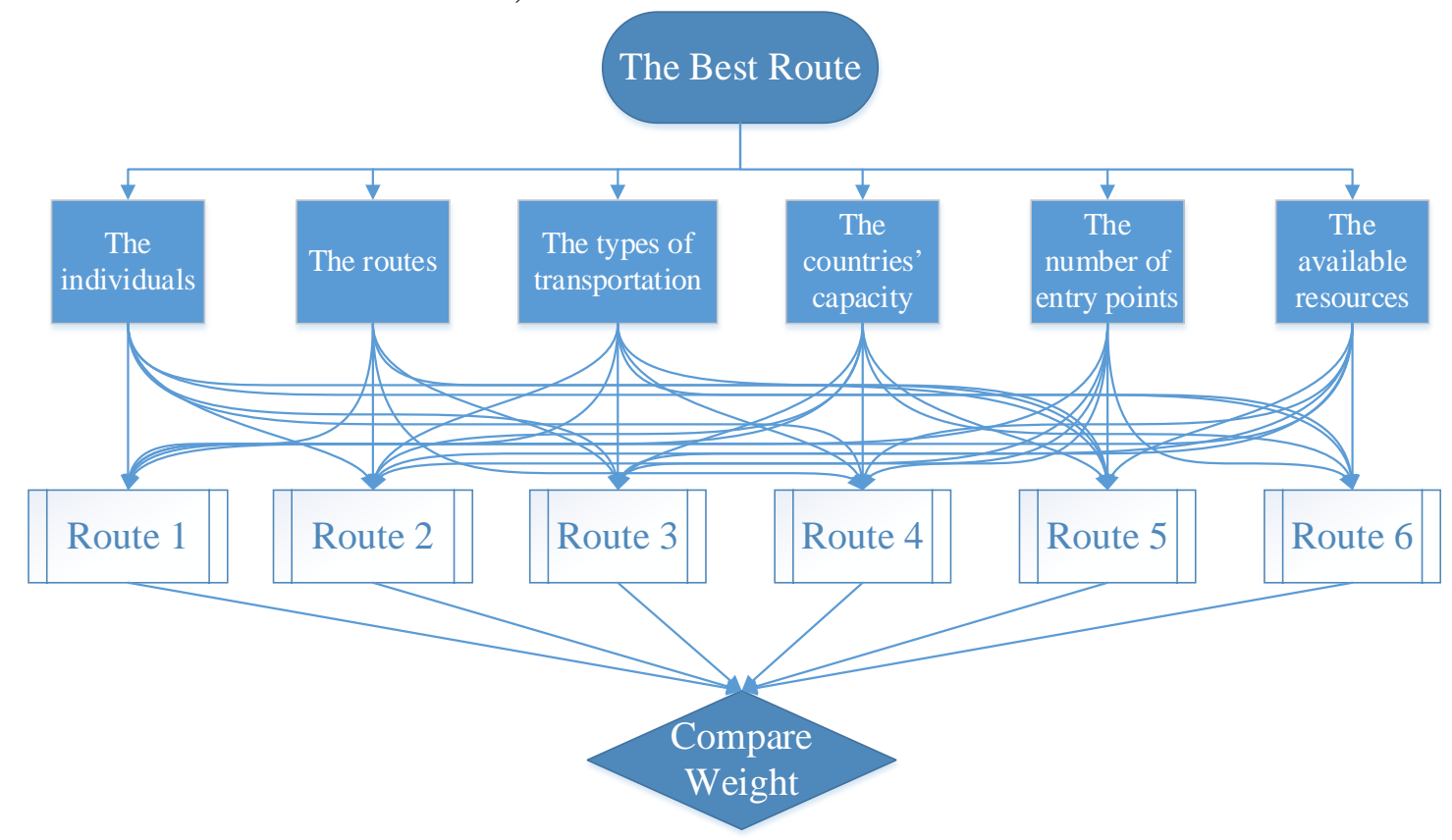

Figure1. The hierarchical structure model of the refugee crisis analysis

\section{- The Test of Total Level Sorting and Consistency}

Calculated by the corresponding formula, we can get the consistency ratio level of total order stochastic:

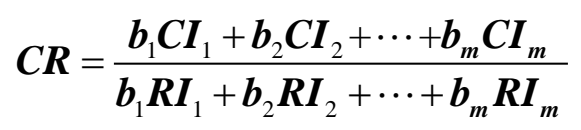

The weight of the total target of route $C_{1}, C_{2}, C_{3}, C_{4}, C_{5}, C_{6}$ is:

$$
\{0.115,0.128,0.29,0.37,0.048,0.049\}
$$

Moreover, $\boldsymbol{C R}=0.0945<0.1$, so the level of total order passes the consistency test.

\section{- The Establishment of Minimum cost Maximum flow Algorithm}

We regard the European and the origin of refugees as the network apex, and use the minimum cost maximum flow algorithm to solve the flow of refugees. The route is as follows:
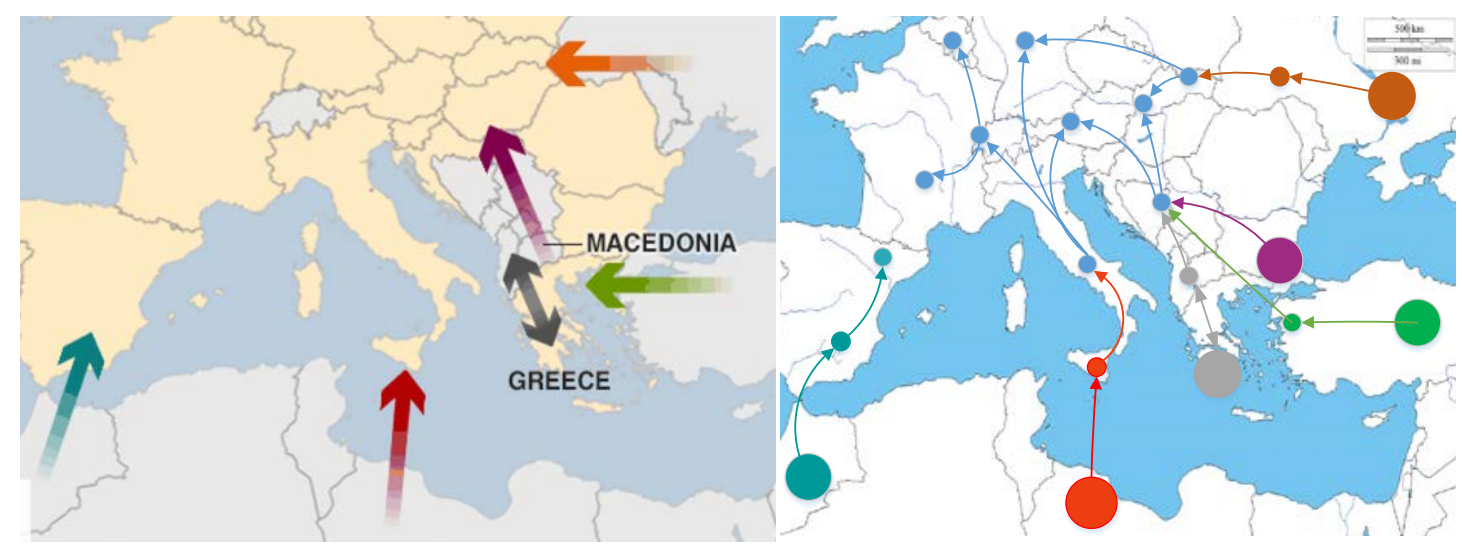

Figure 2. The Route of Flow of Refugees 
- Results of the AHP Model

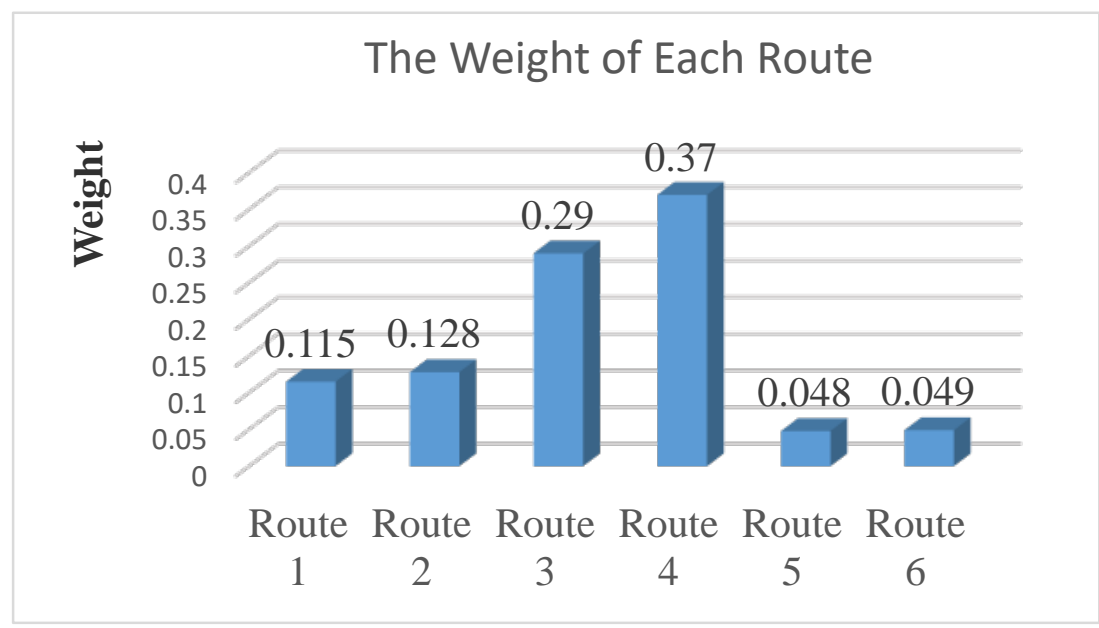

Figure 3. The weights of six routes

From the above chart, using the arithmetic average method, we can get the weights of each scheme C_4>C_3 $>$ C_2 $>$ C_1 $>$ C_6 $>$ C_5, ,so the total weight of Route4 is the largest.

\section{- The Results of Minimum cost Maximum flow Algorithm}

We can get the flow route of refugee by the minimum cost maximum flow algorithm. We can see that the Mediterranean route is still the most popular route, and the strategy I is the best. Not only the number of refugee casualties reached the minimum, but the flow rate is also satisfactory.

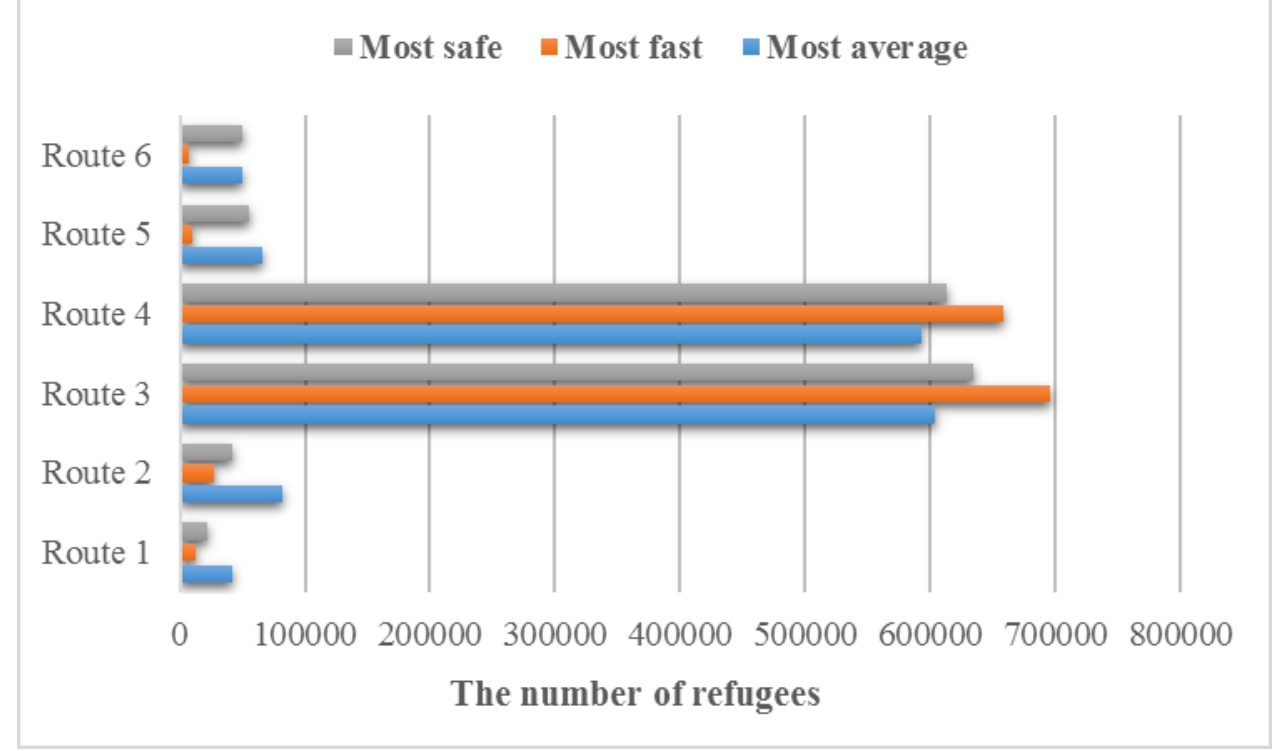

Figure 4. The number of refugees of each route

\section{Dynamic analysis model of resource}

\section{Analysis Approach.}

For limited resources allocation, if we achieve the optimal number of resettled refugees, or make the time over placing refugees shortest, etc. it means that the resources is effective use. If only a quantitative evaluation target, the resource allocation problem can be solved by the dynamic programming method. Nonetheless, like refugee resettlement, we need to take quantitative qualitative objectives into consideration. As a result, Multi - objective Fuzzy Optimization and Dynamic Planning Analysis is used to achieve the allocation of limited resources, making refugee resettlement and resource allocation optimal. In the current refugee resettlement program, refugees are arranged in 
cities discretely, and the government sets up refugee settlements, distribute materials, and give medical care, and so on.

\section{Model Establishing and Solving.}

For limited resources allocation, if we achieve the optimal number of resettled refugees, or make the time over placing refugees shortest, etc. it means that the resources is effective use. If only a quantitative evaluation target, the resource allocation problem can be solved by the dynamic programming method. Nonetheless, like refugee resettlement, we need to take quantitative qualitative objectives into consideration. As a result, Multi - objective Fuzzy Optimization and Dynamic Planning Analysis is used to achieve the allocation of limited resources, making refugee resettlement and resource allocation optimal. In the current refugee resettlement program, refugees are arranged in cities discretely, and the government sets up refugee settlements, distribute materials, and give medical care, and so on.

\section{1) Establish fuzzy optimization dynamic programming model}

Now let $a=\left(a_{1}, a_{2}, \cdots, a_{l}\right)^{T}, d_{j}=\left(d_{1 j}, d_{2 j}, \cdots, d_{w j}\right)^{T},(j=1,2, \cdots, n)$

The process of allocating resources to a resource allocation site as a stage.

Phase variable $k$ : It indicates the allocation of resources to the $\mathrm{k}$-th site, $k=1,2, \cdots, p$,

State variables

$$
S_{k}=\left(S_{1 K}, S_{2 K}, \cdots S_{w K}\right)^{T}
$$

Where $S_{l k}$ represents the $l-t$ th resource assigned to the $k$-th to the $p$-th site $(l=1,2, \cdots, w)$.

State transition equation: $S_{K+1}=S_{K}-X_{K},(\mathrm{~K}=1,2, \cdots, p)$

Allow decision set: $\mathrm{D}_{K}\left(\mathrm{~S}_{K}\right)=\left\{\left(x_{1 k}, x_{2 k}, \cdots, x_{w k}\right)^{T} \mid 0 \leq x_{l k} \leq S_{l k}, X_{K} \subset d_{j}\right\}$

Phase target $v_{k}\left(X_{k}\right)$ : Optimal size decision allocated to site $\mathrm{K}$ from resource owned $S_{K}$

Set $X_{K}=d_{j}$, by the fuzzy optimization model, there is

$$
v_{k}\left(X_{k}\right)=\frac{1}{1+\left(\frac{1-W_{\bullet K K}^{T} R_{\bullet j}}{W_{\bullet K K}^{T} R_{\bullet j}}\right)^{2}} \quad j=1,2, \cdots, n
$$

Where: $W_{\bullet K}$ is an index weight vector of the k-th relief sites

${ }_{K} R_{\bullet} j$ is J-th column vector index relative membership degree matrix of the k-th relief sites.

Optimal value function $f_{K}\left(S_{K}\right)$ : Refers to the quantity of $S_{K}$ resource allocation for the construction of the $k$ relief site to the $p$ site relief when take optimal strategy decision of relative optimal membership degree maximum. The recurrence relation of multi objective fuzzy optimization dynamic programming is

$$
\left\{\begin{array}{c}
f_{K}\left(S_{K}\right)=\max _{X_{K} \subset D_{K}\left(S_{K}\right)}\left\{v_{K} X_{K}+f_{K+1}\left(\mathrm{~S}_{K+1}\right)\right\} \quad k=p-1, \cdots, 1 \\
f_{p}\left(\mathrm{~S}_{P}\right)=\max _{X_{P}=S_{P}} \mathrm{~V}_{P}\left(\mathrm{X}_{P}\right)
\end{array}\right.
$$

Corresponding to $f_{K}\left(S_{K}\right)$ decision is the best decision stage $\mathrm{K}$, denoted $X_{k}^{*} \quad k=p, p-1, \cdots, 2,1$

\section{2)Results of the Model}

First of all, we give a definition of the allocation degree $\left(D=\frac{S_{l k} \times W}{\max S_{l k}}\right)$ to measure the allocation of a resource at a certain time. The closer to 1 , the more efficient is the allocation. Using the formula in 
the model, taking 15 days for the step, we calculate the allocation of degree of state capacity, medical aid and food supply involving government merely, and draw corresponding change curves. The result is shown in the following picture:

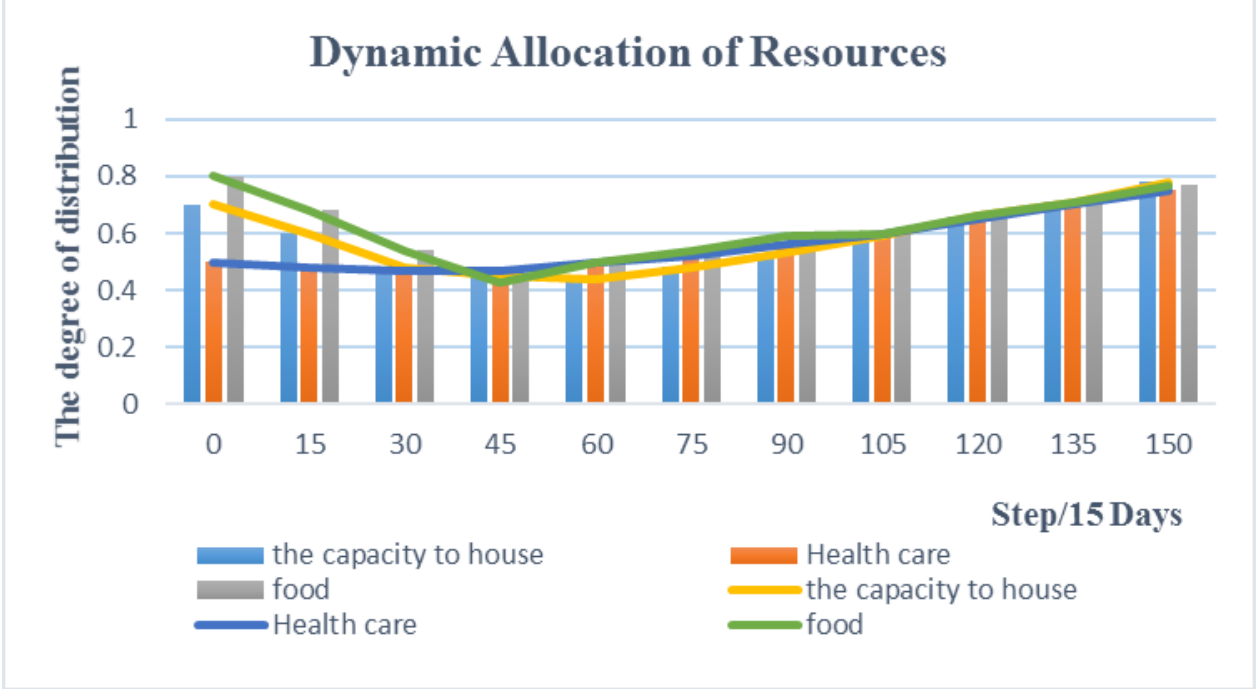

Figure 5. The dynamic allocation of resources of government

Next, we added artificial resources provided by non-governmental institutions, again to 15 days for the step, recalculated the Distribution degree of the country's capacity, medical aid and food supplies to and put together into an array of curve. The distribution of the histogram shows that after the refugees arrived in Europe, the first element is the accommodation and food to survive. In addition, we found that with the government and non-governmental institutions of aid, the volatility of the living conditions of the refugees is smaller than no non-governmental institutions involved.

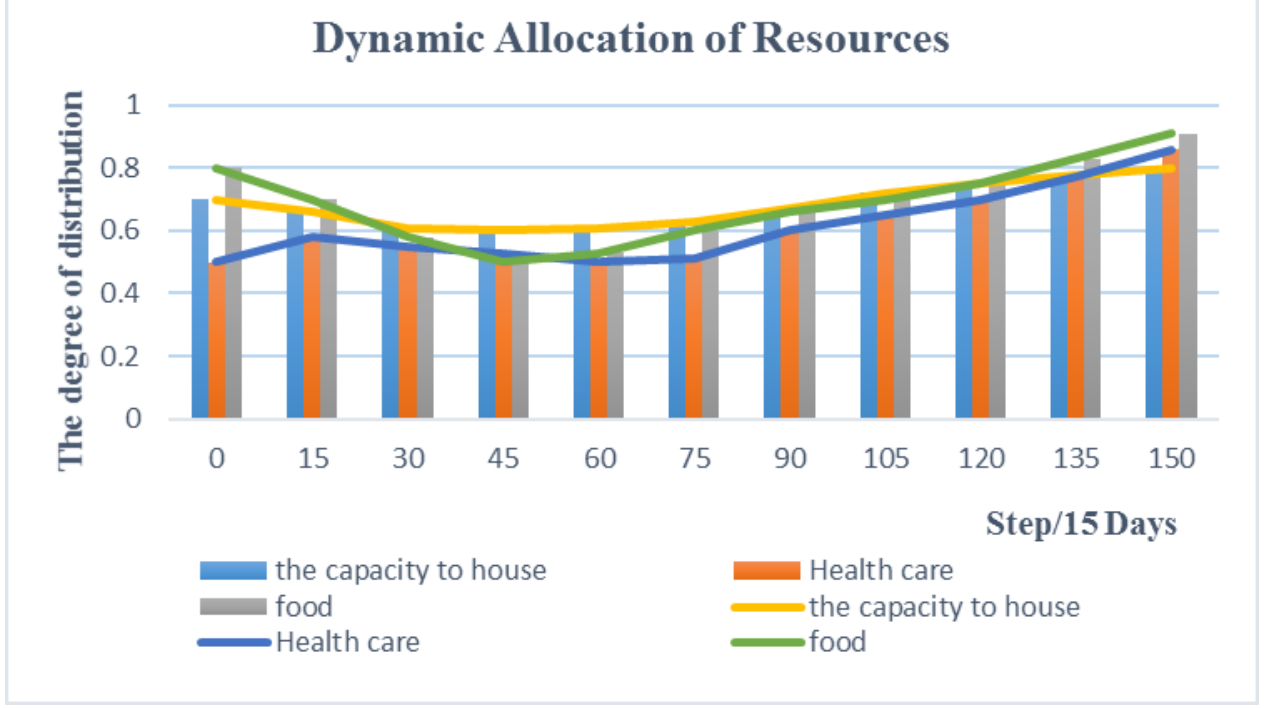

Figure 6. The government and the non-governmental relationship

\section{Summary}

Analytic Hierarchy Process is a great measure of the refugee crises, determining the number of refugees, as well as the rate and point of entry necessary to accommodate their movement. Then, we use the min-cost max-flow of algorithm to obtain the optimal route of refugee migration. Dynamic resource allocation model can usefully allocate resources reach to each shelter, enabling the refugees to be effectively placed. When considering external events and scalability issues, the model also reasonably achieves the optimal route of refugee migration. 
With our research, we can come up with an effective solution regarding the refugee migration, and recommendations to the United Nations, which help solve the problems and maintain the maintenance of world peace and development.

\section{References}

[1]. European migrant crisis. Wikipedia: https://en.wikipedia.org/wiki/European_migrant_crisis

[2]. Althaus, Refugee crisis in Europe: structure, cause and effect analysis[J].

[3]. Kay Hailbronner, Comparative Law of Asylum and Immigration in Europe [J].

[4]. Ban Ki-moon. Opening Remark at UNRWA High-Level Meeting [Z]. September 24, 2009.

[5]. The 2015 Refugee Crisis in the European Union Elspeth Guild, Cathryn Costello, Madeline Garlick and Violeta Moreno-Lax[R].CPES: No. 332, September 2015.

[6]. Dimitri Fazito, Christopher McCarty. Network Analysis of the Contemporary "International Re-fugee System": is there any structure?[J].

[7]. Turkey, the Syrian Refugee Crisis and the Changing Dynamics of Transit Migration [J].

[8]. Carmeli A. Top Management Team Behavioral Integration and the Performance of Service Organizations.[J]. Culture \& Society.

[9]. Charles Westin, Migration Branch [J]. INTERNATIONAL MIGRATION (PAPERS 34).

[10]. Anja Wiesbrock(2011). The Integration of Immigrants in Sweden: a Model for the European Union [J]? INTERNATIONAL MIGRATION.

[11]. The sea route to Europe: The Mediterranean passage in the age of refugees [R].EU:UNHCR, 1 July 2015.

[12]. Migrant crisis: Migration to Europe explained in graphics. BBC, 2015: http://www.bbc.com/news/world-europe-34131911

[13]. International Organization for Migration. http://www.iom.int/

[14]. UNHCR. http://www.unhcr.org/

[15]. MIGRATION FLOWS - EUROPE. http://migration.iom.int/europe/ 